\title{
DEVELOPMENT OF A THREE-DIMENSIONAL MODEL OF THE ATMOSPHERIC BOUNDARY LAYER USING THE FINITE ELEMENT METHOD
}

\author{
R. L. Lee \\ P. M. Gresho
}

November 22, 1977

Work perfornted under the auspices of the U.S. Department of Energy by the UCLLL. under contract number W-7405-ENG-48.

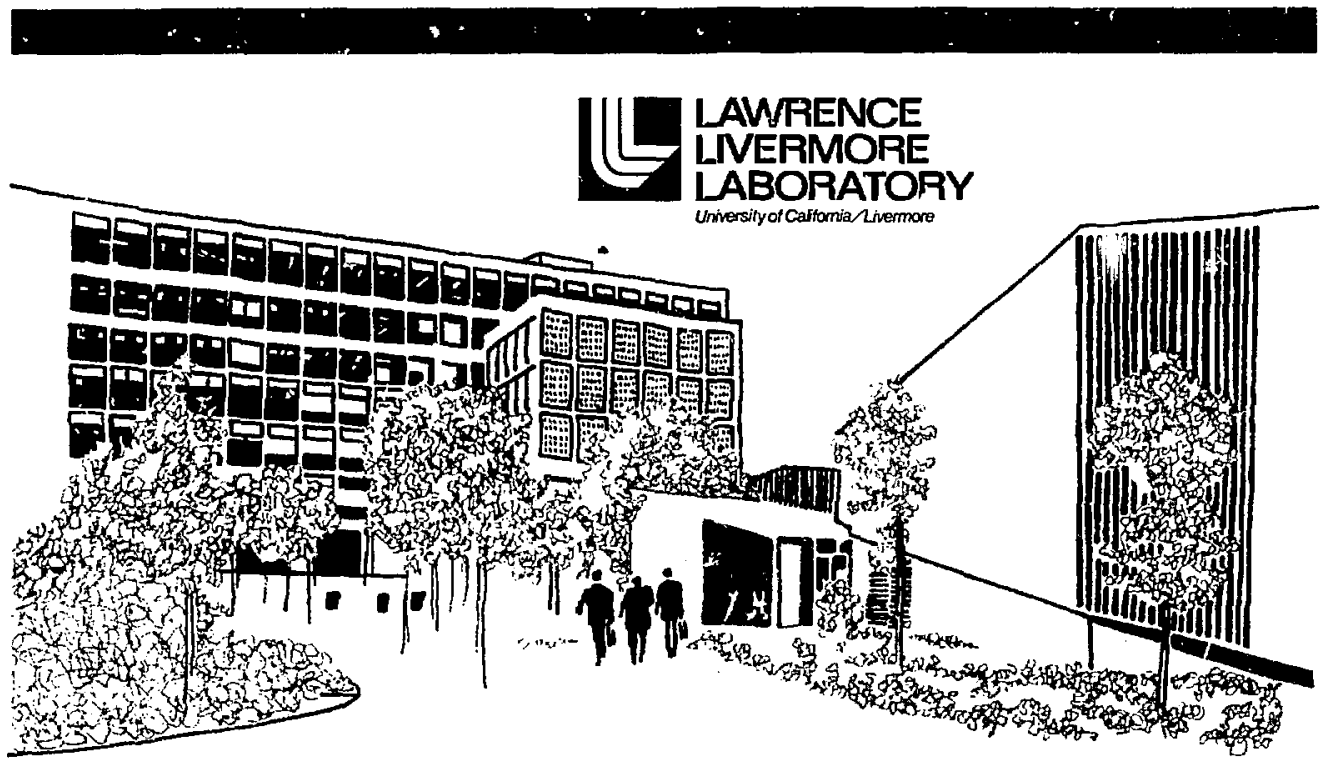




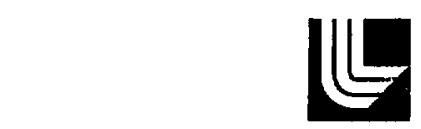

LAWRENCE LNERMORE LABORATORY

Univorsity of Caltomia/Livormono, Calitornia/94550

UCRL-52366

\title{
DEVELOPMENT OF A THREE-DIMENSIC NAL MODEL OF THE ATMOSPHERIC BOUNL ARY LAYER USING THE FINITE ELEMENT METHOD
}

\author{
R. L. Lee
}

P. M. Gresho

M. S. date: November 22, 1977

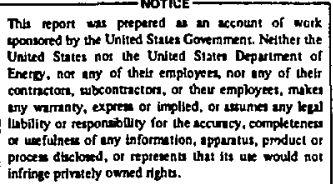

infringe primitely owned right. 


\section{CONTENTS}

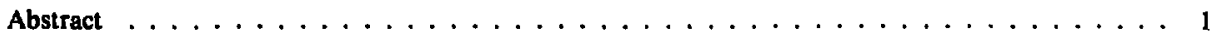

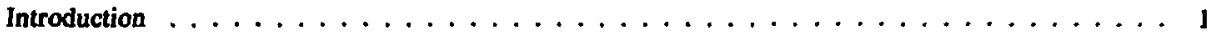

Basic Equations for Atmospheric Boundary Layer Model . . . . . . . . . . . . . . . . 2

The Navier-Stokes Equations . . . . . . . . . . . . . . . . . . . . . 3

The Galerkin Equations ..............................4

The Finite Element Matrices ..............................5

Solution Procedure .................................8

Storage Considerations and Linear Equation Solvers . . . . . . . . . . . . . . . . . . 13

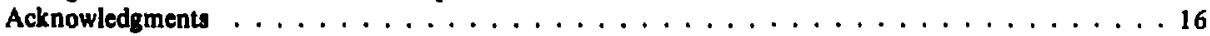

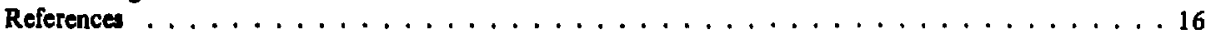




\section{DEVELOPMENT OF A THREE-DIMENSIONAL MODEL OF THE ATMOSPHERIC BOUNDARY LAYER USING THE FINITE ELEMENT METHOD}

\section{ABSTRACT}

This report summarizes our current effort and ideas toward the development of a model for the planetary boundary layer using the finite element technique. As an initial step, the finite element methodology is applied to simpler version of the boundary layer equations given by the two-dimensional, constant-property, incompressible conservation equations (Navier-Stokes equations). Solution procedures for both the steady-state and transient equations are discussed. For the transient problem, a variable time-step, trapezoid-rule algorithm with dynamic time-truncation error control is presented. The resulting system of nonlinear algebraic equations is solved by a Newton iteration procedure with a frontal solution scheme used for the linear set of equations. The need to develop a suitable linear equation solver, with respect to minimization of computer storage and execution costs, particularly for large (three-dimensional) finite element problems, is also discussed.

\section{INTRODUCTION}

For several years we worked toward developing a dynamic (predictive) model of the planetary boundary layer (PBL). Our goal is to achieve two- and three-dimensional computer codes using the finite element method (FEM). This method can provide accurate simulations and predictions of the winds, temperature, humidity, and pollutant concentrations in the lowest few kilometers of the atmosphere over horizontal domains ranging from several to several hundred kilometers.

We began FEM modeling by developing one-, two-, and three-dimensional codes for solving the advection-diffusion equation (with prescribed velocities). These codes formed the basis for the next step: the solution of the two-dimensional, Navier-Stokes equations. At present, we have the following operational codes for incompressible flows:

- Steady-state, isothermal, Navier-Stokes equations.

- Time-dependent, isothermal, Navier-Stokes equations.

- Steady-state, nonisothermal (Boussinesq), Navier-Stokes equations.

Future plans include:

- Conversion of the two-dimensional, Navier-Stokes codes to PBL codes by adding appropriate, nonlinear, eddy diffusion coefficients (turbulence parameterization) and Coriolis effects.

- Application of these codes to typical PBL flow simulations (e.g., stratified shear flow over complex terrain).

- Extension of two-dimensional codes to three dimensions.

The last task is the most difficult and may depend on the development of new and powerful iterative solution methods for solving systems of algebraic equations.

This report summarizes the mathematical techniques used in our two-dimensional, Navier-Stokes codes and presents plans and problems associated with three-dimensional computations. 


\section{BASIC EQUATIONS FOR \\ ATMOSPHERIC BOUNDARY LAYER MODEL}

We plan to apply the Galerkin finite element method (FEM) using either linear or quadratic isoparametric elements to integrate the three-dimensional, time-dependent equations describing flow and temperature in the atmospheric boundary layer. The basic dynamical equations for the model are the three equations of motion, the continuity equation, and the energy equation. Additional equations describing the transfer of water vapor and pollutante (both reactive and inert) are initially excluded from the current discussion but are being considered for the final model.

With the usual notation, the governing equations of motion are (in an Eulerian reference frame):

$$
\begin{aligned}
& \frac{D u}{D t}-f v+\frac{1}{\rho} \frac{\partial p}{\partial x}=K \nabla_{H}^{2} u+\frac{\partial}{\partial z}\left(K_{M} \frac{\partial u}{\partial z}\right), \\
& \frac{D v}{D t}+f u+\frac{1}{\rho} \frac{\partial p}{\partial y}=K \nabla_{H}^{2} v+\frac{\partial}{\partial z}\left(K_{M} \frac{\partial v}{\partial z}\right),
\end{aligned}
$$

and

$$
\frac{D_{W}}{D t}+g+\frac{1}{\rho} \frac{\partial p}{\partial z}=K \nabla_{H}^{2} w+\frac{\partial}{\partial z}\left(K_{M} \frac{\partial w}{\partial z}\right),
$$

where the Lagrangian derivative $D / D t$ is given by

$$
\frac{D}{D t}=\frac{\partial}{\partial t}+V \cdot \nabla
$$

and

$$
\nabla_{H}^{2}=\frac{\partial^{2}}{\partial x^{2}}+\frac{\partial^{2}}{\partial y^{2}} .
$$

-and the velocity $\mathrm{V}$ is given in component form by $\mathrm{V}=(\mathrm{U}, \mathrm{v}, \mathrm{w})$. The Coriolis parameter $\mathrm{f}$ and horizontal eddy diffusivity $\mathrm{K}$ are constants and $\rho$, the density, is either constant or a prescribed function of the height coordinate $\mathbf{z}$.

The (incompressible) continuity equation is

$$
\boldsymbol{\nabla} \cdot \mathrm{v}=\mathbf{0}
$$

and the energy equation is

$$
\frac{D \theta}{D t}=K_{H} \nabla_{H}^{2} \theta+\frac{\partial}{\partial z}\left(K_{H} \frac{\partial \theta}{\partial z}\right),
$$

where $\theta$ is the (potential) temperature. Although we do not yet include any heat source (or sink) terms, additional terms representing ffects of radiation and cloud physics will be included later.

In the preceding set of equations, nonlinearities are due to the advection (e.g., $v . V$ ) term and the vertical eddy diffusion coefficients for momentum $\left(\mathrm{K}_{\mathrm{M}}\right)$ and heat $\left(\mathrm{K}_{\mathrm{H}}\right)$ transfer. Typically, these eddy coefficients are functions of the wind shear $\left(\partial \mathrm{\partial U} / \mathrm{\partial z}=\left[(\mathrm{\partial u} / \mathrm{\partial z})^{2}+(\partial \mathrm{av} / \mathrm{\partial z})^{2}\right]^{1 / 2}\right)$ and vertical temperature gradient $(\partial \theta / \partial z)$ with 
the exact form being dependent on the particular "K theory" model used. For example, a model of the eddy coefficient for momentum is given by

$$
K_{M}=K(h) \exp \{-a(z-h)\}+\widetilde{K}[1-\exp \{-a(z-h)\}] ; h \leqslant z \leqslant H
$$

where

$$
\begin{aligned}
\widehat{R} & =R^{2}\left[\left(\frac{\partial u}{\partial z}\right)^{2}+\left(\frac{\partial v}{\partial z}\right)^{2}\right]^{1 / 2} S_{M} ; \alpha=1 / 100 . \\
K(h) & =K(z=h)=\text { constant; } H=\text { Atm. boundary layer height } ; h=\text { Constant flux layer height. } \\
S_{M} & =\left\{\begin{array}{l}
(1+: R i)^{-2}, \quad R i>0 \\
(1-3 R i)^{2}, \quad R i<0
\end{array}\right. \\
1 / R= & 1 / 0.00027 u_{g} / f+1 / k z ; f, u_{g}, k \text { are constants. } \\
R i= & \frac{g \frac{\partial \theta}{\partial z}}{\theta_{0}\left(\frac{\partial U}{\partial z}\right)^{2}} \text { (Richardson number). }
\end{aligned}
$$

In addition to the governing equations, two other pieces of information are needed for the solution:

- Initial conditions. The initialization of the model with real data is a nontrivial problem, particularly where measurement stations providing wind and temperature data are scarce. Mathematically, the initial conditions for velocity must at least satisfy mass conservation [Eq. (4)] over the domain of computation. A violation of this requirement will lead to startup problems resulting in severe shock conditions at the initial instant of integration. We will deal with initialization of the model as a separate problem and, for the purpose of this discussion, will assume that the specified initial conditions are compatible with the calculative procedure.

- Boundary conditions. For our calculations, a typical computational domain consists of straight-sided top and lateral boundaries with a bottom topography that is variable and prescribed. At the bottom boundary, we apply the usual no-slip condition for the velocities and prescribe a linear combination of the surface temperature and heat flux. Inflow conditions (velocity, temperature) are also prescribed at lateral inflow boundaries. We plan to test several types of outflow conditions to determine which is most appropriate for our use.

\section{THE NAVIER-STOKES EQUATIONS}

Instead of starting with the full three-dimensional, planetary layer equations, we began by considcring the two-dimensional, Navier-Stokes equati ins. The main simplifications associated with this set of twodimensional equations are constant properties $\left(\rho, K, K K_{M}\right)$, isothermal (no energy equation), and no Coriolis terms. With these simplifications, the equations can be written (converting to tensor notation and the summation convention) as

$$
\rho \frac{\partial \hat{u}_{i}}{\partial t}+\rho \hat{u}_{j} \frac{\partial \hat{u}_{i}}{\partial x_{j}}-\frac{\partial \tau_{i j}}{\partial x_{j}}=0
$$




$$
\frac{\partial \hat{u}_{i}}{\partial x_{i}}=0
$$

with

$$
\tau_{i j}=-\hat{p} \delta_{i j}+K\left(\frac{\partial \hat{u}_{i}}{\partial x_{j}}+\frac{\partial \hat{u}_{j}}{\partial x_{i}}\right) .
$$

$\mathrm{K}$ is the (constant) viscosity.

There are severc" theoretically equivalent formulations of the Navier-Stokes equations. The preceding formulation has been used in most finite element calculations because the stress terms $\left(\tau_{i j}\right)$, when integrated by parts according to the Galerkin procedure, lead to natural boundary conditions involving surface tractions. It appears that traction-type boundary conditions may be particularly appropriate for simulations of outflow situations. Another alternative formulation, used extensively in finite difference calculations, replaces the continuity equation with a Poisson equation for the pressure given by

$$
\nabla^{2} \hat{p}=-\rho\left\{\left(D^{2}+\hat{v} \cdot \nabla D\right)+2\left(\frac{\partial \hat{v}}{\partial x} \frac{\partial \hat{u}}{\partial y}-\frac{\partial \hat{\hat{u}}}{\partial x} \frac{\partial \hat{v}}{\partial y}\right)\right\}-\rho \frac{\partial D}{\partial t}+K \nabla^{2} D,
$$

where

$$
D=\nabla \cdot \hat{v}
$$

It is important to retan certain $D$ terms in the preceding equation, since the discretized form of $D$ is only approximately zero.

In this formulation, the velocity and pressure are treated as if they were decoupled; therefore, a solution requires alternatively solving for pressure and velocity from Eqs. (9) and (6). Here, one difficulty lies in setting the boundary conditions for pressure. In general, velocities rather than pressures are known at boundaries; therefore, one has to obtain the more difficult Neumann boundary conditions for pressure indirectly by imposing Eq. (6) at the boundary. This is especially cumbersome with the FEM formulation, since the boundary conditions require second derivatives of the velocity. This feature, plus the appearance of derivatives of third order in Eq. (9) precl.des the use of low-order $\left(\mathrm{C}^{\circ}\right)$ basis functions to approximate velocities in the finite element procedure. Hence, we employ Eqs. (6), (7), and (8) in our FEM formulation.

\section{THE GALERKIN EQUATIONS}

The approximation of a set of partial differential equations as given in Eqs. (6) and (7) by the Galerkin procedure is straightforward. We assume th: velocity and pressure fields are approximated by

$$
\hat{u}_{i}\left(x_{\ell}, t\right)=\sum_{i=1}^{N} \phi^{I}\left(x_{\ell} ; u_{i}^{l}(t)=\phi^{I} u_{i}^{l} ; \quad(i, \ell=1,2)\right.
$$

and

$$
\hat{p}\left(x_{i}, t\right)=\sum_{\alpha=1}^{. M} \psi^{\alpha}\left(x_{i}\right) p^{\alpha}(t)=\psi^{\alpha} p^{\alpha} ;(i=1,2)
$$

where $\phi^{\prime}$ and $\psi^{\alpha}$ are sets of known basis functions, and $u_{i}^{\prime}, p^{\alpha}$ are the nodal unknowns to be solved for as a function of time. It is important, however, as is indicated above and discussed later, that we allow for a different set of basis functions for pressure than velocities. 
Substituting the approximations for $\hat{\mathrm{u}}_{\mathrm{i}}$ and $\hat{\mathrm{p}}$ as given by Eqs. (10) and (11), into Eqs. (6) and (7), multiplying by the appropriate weighting functions, and integrating the stress term by parts, we obtain the following set of ordinary differential equations (ODE's) for the amplitude coefficients:

$$
\begin{aligned}
& {\left[\int_{\Omega} \rho \phi^{\mathrm{J}} \phi^{\mathrm{J}} \mathrm{d} \phi\right] \frac{\mathrm{du} \mathrm{u}_{\mathrm{i}}^{\mathrm{J}}}{\mathrm{dt}}+\left[\int_{\Omega} \rho \phi^{\mathrm{l} \phi^{\mathrm{L}} \mathrm{u}_{\mathrm{j}}^{\mathrm{L}}} \frac{\partial \phi^{\mathrm{J}}}{\partial \mathrm{x}_{\mathrm{j}}} d \boldsymbol{\alpha}\right] \mathrm{u}_{\mathrm{i}}^{\mathrm{J}}} \\
& +\left[\int_{\Omega} K \frac{\partial \phi^{\mathrm{I}}}{\partial \mathrm{x}_{\mathrm{j}}} \frac{\partial \phi^{\mathrm{J}}}{\partial \mathrm{x}_{\mathrm{j}}} \mathrm{d} \boldsymbol{\theta}\right] \mathrm{u}_{\mathrm{i}}^{\mathrm{J}}+\left[\int_{\Omega} \mathrm{K} \frac{\partial \phi^{\mathrm{I}}}{\partial \mathrm{x}_{\mathrm{j}}} \frac{\partial \phi^{\mathrm{J}}}{\partial \mathrm{x}_{\mathrm{i}}} \mathrm{de} \boldsymbol{\mathrm { u } _ { \mathrm { j } } ^ { \mathrm { J } }}\right. \\
& -\left[\int_{\Omega} \frac{\partial \phi^{l}}{\partial x_{i}} \psi^{\alpha} d \alpha\right] p^{\alpha}=\int_{\partial \Omega} \phi^{l} \tau_{i j} n_{j} d \ell ;(i, j=1,2 ; I, J, L=1,2, \ldots, N) \\
& {\left[-\int_{\Omega} \psi^{\alpha} \frac{\partial \phi^{I}}{\partial x_{i}} d \mu\right] u_{i}^{I}=0 \quad(\alpha=1,2, \ldots, M)}
\end{aligned}
$$

The (global) set of ODE's can be written symbolically as

$$
\underset{\sim}{\operatorname{Mq}}+[\underset{\approx}{N}(q)+\underset{\sim}{\mathbf{K}}] q \underline{q}=\underset{\sim}{\mathbf{F}}
$$

$$
\begin{aligned}
& \text { where } \underset{\approx}{\mathbb{M}}=\text { mass matrix, } \int_{\Omega} \phi^{l} \phi^{\jmath} d \omega \text {, } \\
& \underset{\approx}{\mathbb{N}}=\text { nonlinear convective matrix, } \\
& \underset{\mathbb{Z}}{\mathbf{K}}=\text { linear matrix of diffusion and pressure terms, } \\
& \underline{F}=\text { forcing function from boundary conditions, } \\
& q=\text { vector of nodal unknowns }(u, v, p) \text {. }
\end{aligned}
$$

\section{THE FINITE ELEMENT MATRICES}

Following the simplest finite element methodology, we select a set of piecewise continuous $\left(\mathrm{C}^{\circ}\right)$ basis functions. The globai integrals are obtained by first performing integrations locally (via Gaussian quadrature) over each individual element, then assembling contributions from every element joined at each node:

$$
\int_{\Omega}(\quad) d \omega=\sum_{1}^{N_{c}} \int_{\Omega_{e}}() d \omega,
$$

where $\mathrm{N}_{\mathrm{e}}$ is the total number of elements. This leads to the following element ievel matrices fo: the nodal uriknowns $\mathrm{g}$ of a single element: 


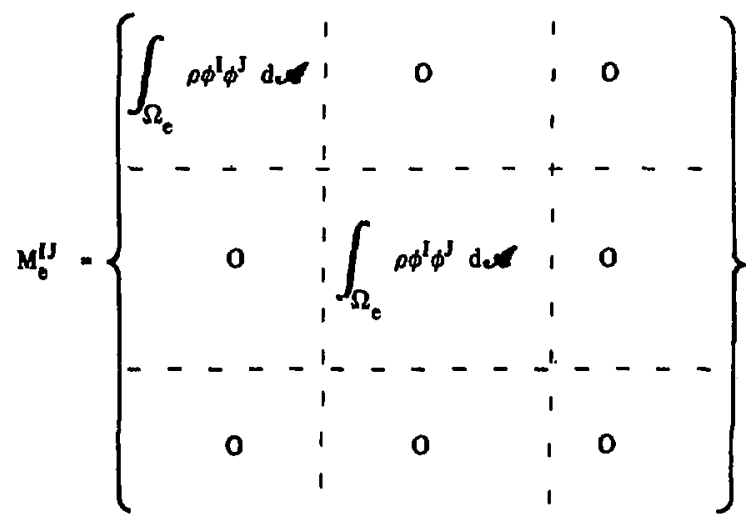

$q^{I}=\left\{u^{I}, v^{I}, p^{I}\right\}^{T}$

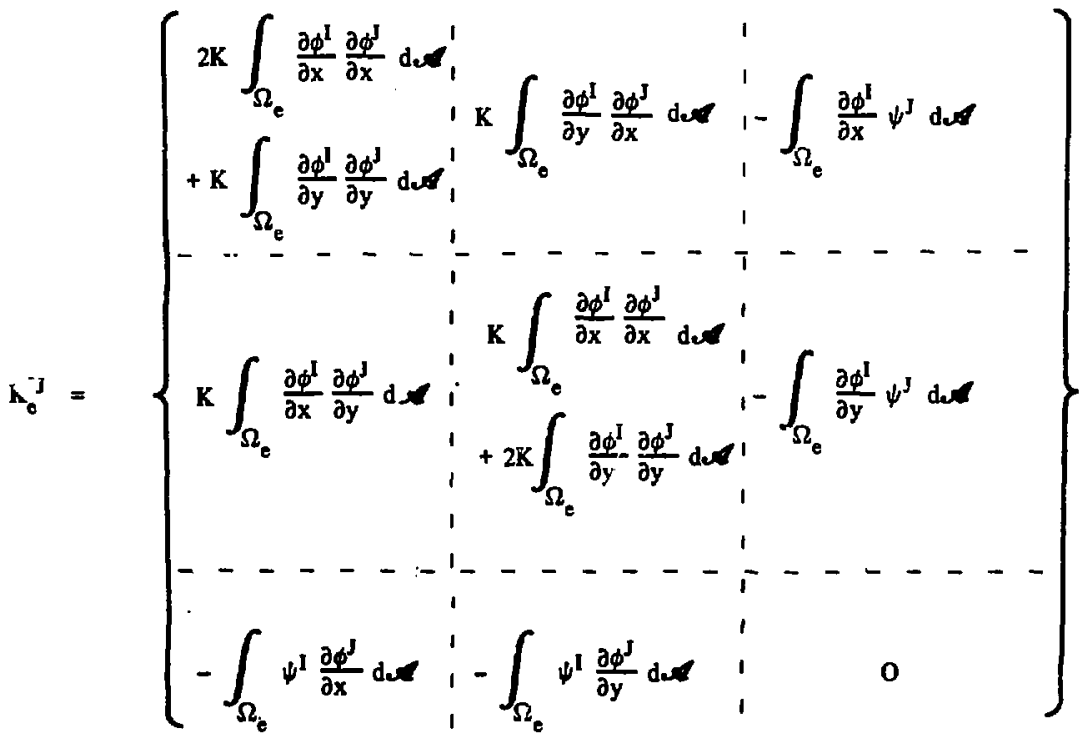


Substituting the approximations for $\hat{u}_{i}$ and $\hat{p}$ as given by Eqs. (10) and (11), into Eqs. (6) and (7), multiplying by the appropriate weighting functions, and integrating the stress term by parts, we obtain the following set of ordinary differential equations (ODE's) for the amplitude coefficients:

$$
\begin{aligned}
& {\left[\int_{\Omega} \rho \phi^{\mathrm{I}} \phi^{\mathrm{J}} \mathrm{d} \omega\right] \frac{\mathrm{du} \mathrm{i}_{\mathrm{i}}^{\mathrm{J}}}{\mathrm{dt}}+\left[\int_{\Omega} \rho \phi^{\mathrm{I}} \phi^{\mathrm{L}} \mathrm{u}_{\mathrm{j}}^{\mathrm{L}} \frac{\partial \phi^{\mathrm{J}}}{\partial \mathrm{x}_{\mathrm{j}}} \mathrm{d} \omega \boldsymbol{u}_{\mathrm{i}}^{\mathrm{J}}\right.} \\
& +\left[\int_{\Omega} K \frac{\partial \phi^{I}}{\partial x_{j}} \frac{\partial \phi^{J}}{\partial x_{j}} d \alpha\right] u_{i}^{j}+\left[\int_{\Omega} K \frac{\partial \phi^{I}}{\partial x_{j}} \frac{\partial \phi^{J}}{\partial x_{i}} d \phi\right] u_{j}^{J} \\
& -\left[\int_{\Omega} \frac{\partial \phi^{l}}{\partial x_{j}} \psi^{\alpha} d \mathcal{L}\right] p^{\alpha}=\int_{\partial \Omega} \phi^{1} \tau_{i j} n_{j} d l ;(i, j=1,2 ; I, J, L=1,2, \ldots, N) \\
& {\left[-\int_{\Omega} \psi^{\alpha} \frac{\partial \phi^{I}}{\partial x_{i}} d \psi\right] u_{i}^{1}=0 \quad(\alpha=1,2, \ldots, M)}
\end{aligned}
$$

The (global) set of ODE's can be written symbolically as

$$
\underset{\sim}{\mathrm{M}}+[\underset{\approx}{\mathbf{N}}(q)+\underset{\approx}{\mathbf{K}}] \underset{\sim}{q}=\underset{\sim}{\mathbf{F}}
$$

$$
\begin{aligned}
& \text { where } \underset{\approx}{\mathbf{M}}=\text { mass matrix, } \int_{\Omega} \phi^{\mathrm{J}} \phi^{\mathrm{J}} \mathrm{d} \boldsymbol{\alpha} \text {, } \\
& \underset{\mathrm{N}}{\approx}=\text { nonlinear convective matrix, } \\
& \underset{\mathrm{K}}{\mathrm{Z}}=\text { linear matrix of diffusion and pressure terms, } \\
& \mathbf{E}=\text { forcing function from boundary conditions, } \\
& q=\text { vector of nodal unknowns }(u, v, p) \text {. }
\end{aligned}
$$

\section{THE FINITE ELEMENT MATRICES}

Following the simplest finite element methodology, we select a set of piecewise continuous $\left(\mathrm{C}^{\circ}\right)$ basis functions. The global integrals are obtained by first performing integrations locally (via Gaussian quadrature) over each individual element, then assembling contributions from every element joined at each node:

$$
\int_{\Omega}() d \omega=\sum_{1}^{N_{e}} \int_{\Omega_{e}}() d \omega .
$$

where $N_{e}$ is the total number of elements. This leads to the following element level matrices for the nodal unknowns $q$ of a single element: 


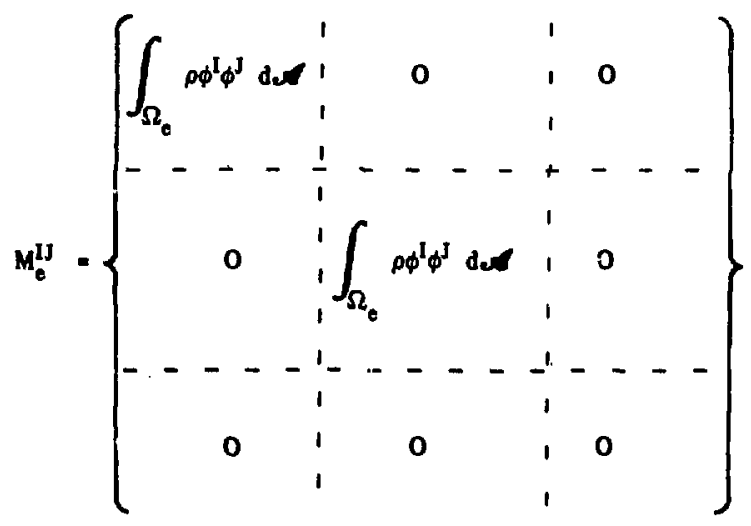

$$
q^{I}=\left(u^{I}, v^{I}, p^{I}\right\}^{T}
$$

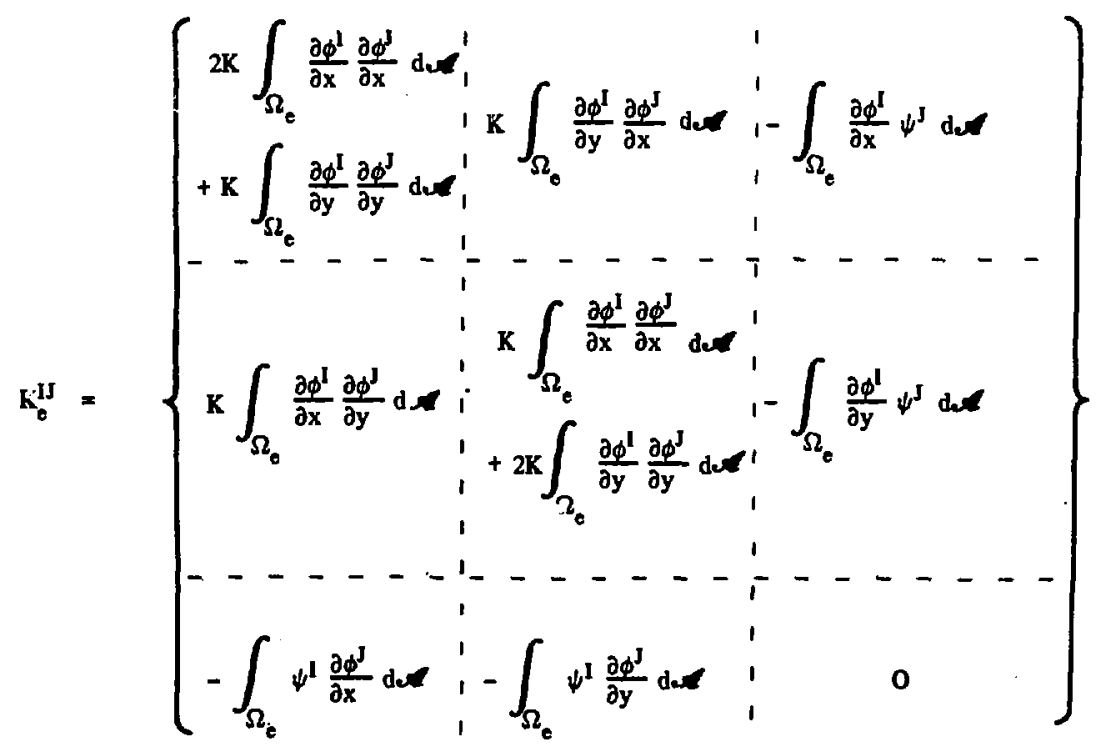




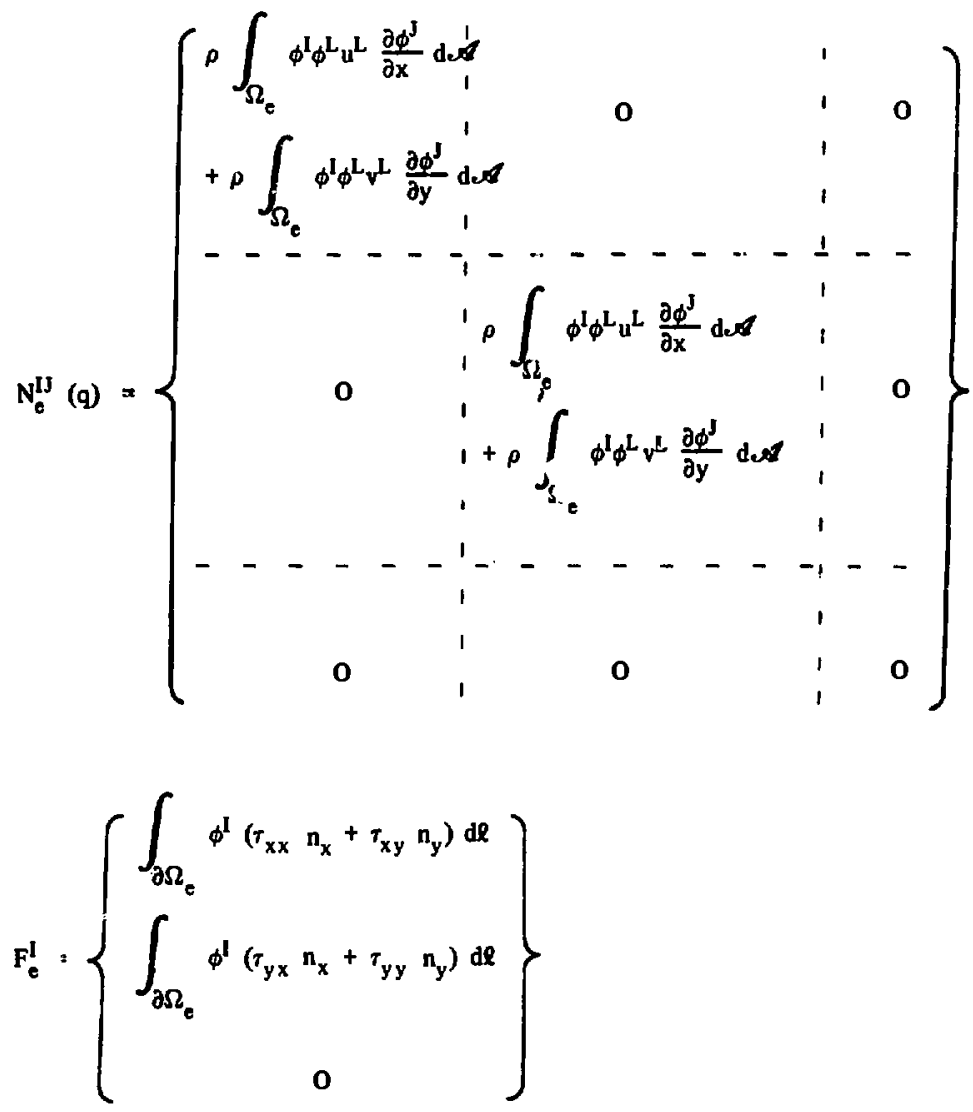

Several observations may be made concerning Eqs. (14) through (19):

1. The set of ODE's in Eq. (14) is time singular; i.e., the equations associated with the incompressibility condition appear as linear algebraic constraints with time derivatives absent.

2. Global matrices are sparse with bandwidths that are small relative to the order of the matrix.

3. The upper two blocks of the mass matrix $M$ is symmetric and positive definite (this property is important for the time-dependent formulation of the equations).

4. The linear matrix $\mathbf{K}$ is symmetric (since the negative of the continuity equation is used) but indefinite.

5. There are no derivatives of the basis functions for pressurc; hence, the continuity constraint or pressure may be relaxed (from $\mathrm{C}^{\circ}$ ), and piecewise discontinuous basis functions may be employed.

A variety of choices involving combinations of basis functions is possible. For the primitive variable $(u, v, p)$ approach, it has been established that error consistency reasons dictate a choice of mixed interpolation, in which the pressure basis functions are chosen to be at least one order lower than velocity basis functions. There is a general concensus that basis functions described by low order polynomials are optimal; most practioners use quadratic (8- or 9-node) elements for velocities and linear (4-node) elements for pressures. Our recent work in this area ${ }^{1}$ indicates that solutions with the 9 -node element are significantly more accurate than those with the 8-node element. 
Anticipating three-dimensional problems, it is possible, however, that even the quadratic element (27node, in this case) would be computationally too expensive, and that only the lowest order interpolation, using linear approximation for velocities with piecewise constants for pressure (8-node, three-dimensional element) may be practical.

These lowest order appruximations, when applied to rectangular meshes, are similar in many ways to finite difference formulations. In fact, our calculations show that this particular element is subject to numerical problems similar in nature to those encountered with staggered-grid, finite difference schemes. A more detailed discussion is given in Reference 1. We are optimistic, however, that these problems may be overcome by using appropriate averaging algorithms recently developed; we will report this aspect in the future.

\section{SOLUTION PROCEDURE}

We obtained solutions (in two-dimensions) to Eq. (14) for both steady-state and transient problems. Steady-state solutions are oblained by either directly attacking the steady-state set of equations [i.e., Eqs. (6) and (7) or Eq. (14) with time derivative terms omitted], or by integrating the time-dependent equations to a sufficiently long time such that transient effects become negligible. At the onset, it is not clear which procedure would be more computationally efficient. We anticipate that the transient procedure, being more generally reliable when employing a variable time-step algorithm (described later), will lead to successful calculations (particularly for higher Reynolds number situations) in a more systematic manner (less guess work required) than the steady-state procedure. The solution techniques employed for each casc are discussed separately.

\section{Solution Techniques for Stendy-State Navier-Stokes Equations}

Upon omission of the time derivative terms, the set of equations to be solved is given by:

$$
\underset{\approx N}{\mathbb{N}}(\mathfrak{q})+\underset{\approx}{\mathbf{K}}] \underset{\sim}{q}=\underset{\sim}{\mathbf{F}}
$$

For the solution of this nonlinear system, we use the Newton-Raphson iteration procedure with analytical evaluation of the Jacobian matrix. By defining $\delta q^{m}=g^{m+1}-q^{m}$, the Newton-Raphson procedure for the $m^{\text {th }}$ iteration is given by

$$
\underset{\approx}{\mathbf{J}^{\mathrm{m}}} \delta \mathrm{g}^{\mathrm{m}}=\mathbf{\sim}-\left[\mathbb{N}\left(\mathrm{q}^{\mathrm{m}}\right)+\underset{\approx}{\mathrm{K}}\right] \mathrm{g}^{\mathrm{m}}
$$

where

$$
\begin{aligned}
& \underset{\approx}{J^{m}}=\frac{\partial}{\partial g}\left[\mathbb{N}\left(q^{m}\right) q^{m}\right]+\underset{\approx}{K} \\
& =\underset{\text { d }}{\mathrm{n} L}+\underset{\mathrm{K}}{\mathrm{K}} \text {. }
\end{aligned}
$$

The nonlinear contribution to $\underset{\approx}{\mathrm{J}}$, conıputed at element level, is given by 


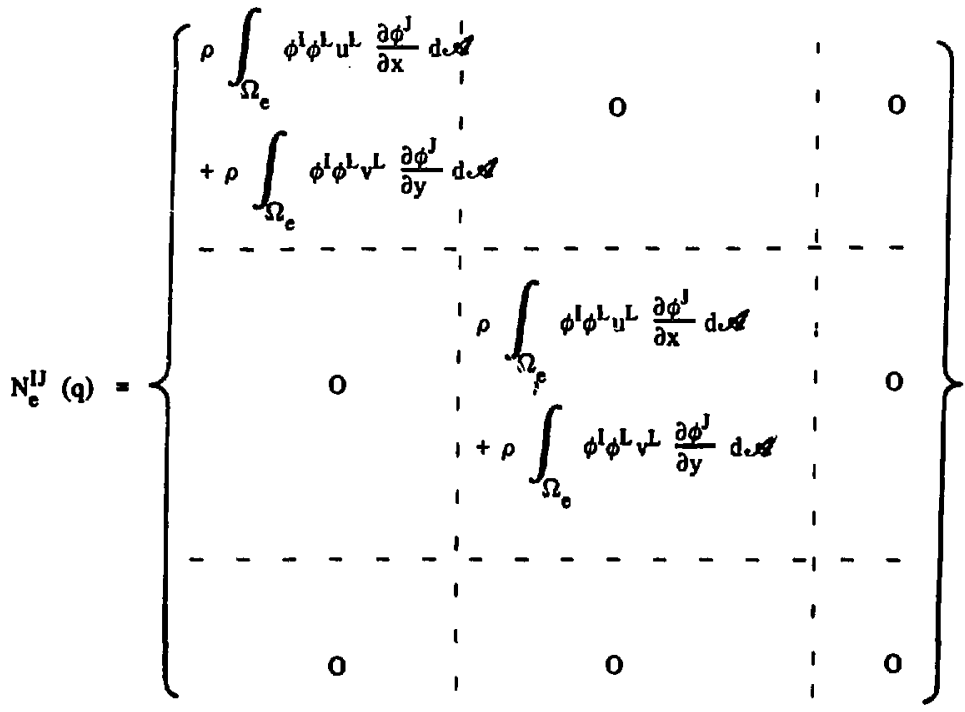

$$
\begin{aligned}
& F_{e}^{I}=\left\{\begin{array}{c}
\int_{\partial \Omega_{e}} \phi^{I}\left(\tau_{x x} n_{x}+\tau_{x y} n_{y}\right) d l \\
\int_{\partial \Omega_{e}} \phi^{I}\left(\tau_{y x} n_{x}+\tau_{y y} n_{y}\right) d l \\
0
\end{array}\right\}
\end{aligned}
$$

Several observations may be made concerning Eqs. (14) through (19):

1. The set of ODE's in Eq. (14) is time singular; i.e., the equations associated with the incompressibility condition appear as linear algebraic constraints with time derivatives absent.

2. Global matrices are sparse with bandwidths that are small relative to the order of the matrix.

3. The upper two blocks of the mass matrix $M$ is symmetric and positive definite (this property is important for the time-dependent formulation of the equations).

4. The linear matrix $K$ is symmetric (since the negative of the continuity equation is used) but indefinite.

5. There are no derivatives of the basis functions for pressure; hence, the continuity constraint or pressure may be relaxed (from $\mathrm{C}^{\circ}$ ), and piecewise discontinuous basis functions may be employed.

A variety of choices involving combinations of basis functions is possible. For the primitive variable $(u, v, p)$ approach, it has been established that error consistency reasons dictate a choice of mixed interpolation, in which the pressure basis functions are chosen to be at least one order lower than velocity basis functions. There is a general concensus that basis functions described by low order polynomials are optimal; most practioners use quadratic (8- or 9-node) elements for velocities and linear (4-node) elements for pressures. Our recent work in this area 1 indicates that solutions with the 9-node element are significantly more accurate than those with the 8-node element. 
Anticipating three-dimensional problems, it is possible, however, that even the quadratic element (27node, in this case) would be computationally too expensive, and that only the lowest order interpolation, using linear approximation for velocities with piecewise constants for pressure (8-node, three-dimensional element) may be practical.

These lowest order approximations, when applied to rectangular meshes, are similar in many ways to finite difference formulations. In fact, our calculations show that this particular element is subject to numerical problems similar in nature to those encountered with staggered-grid, finite difference schemes. A more detailed discussion is given in Reference 1. We are optimistic, however, that these problems may be overcome by using appropriate averaging algorithms recently developed; we will report this aspect in the future.

\section{SOLUTION PKOCEDURE}

We obtained solutions (in two-dimensions) to Eq. (14) for both steady-state and transient problems. Steady-state solutions are obtained by either directly attacking the stendy-state set of equations [i.e., Eqs. (6) and (7) or Eq. (14) with time derivative terms omitted], or by integrating the tin. e-dependent equations to a sufficiently long time such that transient effects become negligisle. At the onset, it is not clear which procedure would be more computationally efficient. We anticipate that the transient procedure, being more generally reliable when employing a variable time-step algorithm (described later), will lead to successful calculations (particularly for higher Reynolds number situations) in a more systematic manner (less guess work required) than the steady-state procedure. The solution techniques employed for each case are discussed separately.

\section{Solution Techniques for Steady-State Navier-Stokes Equations}

Upon omission of the time derivative terms, the set of equations to be solved is given by:

$$
\underset{\approx}{[N(q)}+\underset{\approx}{\mathbf{K}}] \cdot q=\underset{\sim}{\mathbf{F}}
$$

For the solution of this nonlinear system, we use the Newton-Raphson iteration procedure with analytical evaluation of the Jacobian matrix. By defining $\delta q^{m}=q^{m+1}-q^{m}$, the Newton-Raphson procedure for the $m$ th iteration is given by

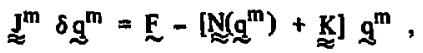

where

$$
\begin{aligned}
& \mathrm{J}^{\mathrm{m}}=\frac{\partial}{\partial \mathrm{q}}\left[\underset{\approx}{N}\left(\mathrm{q}^{\mathrm{m}}\right) \mathrm{g}^{\mathrm{m}}\right]+\underset{z}{\mathbf{K}} \\
& =\underset{\approx N}{\mathrm{~m}}+\underset{\approx}{\mathrm{E}} \text {. }
\end{aligned}
$$

The nonlinear contribution to $\mathrm{J}$, computed at element level, is given by 


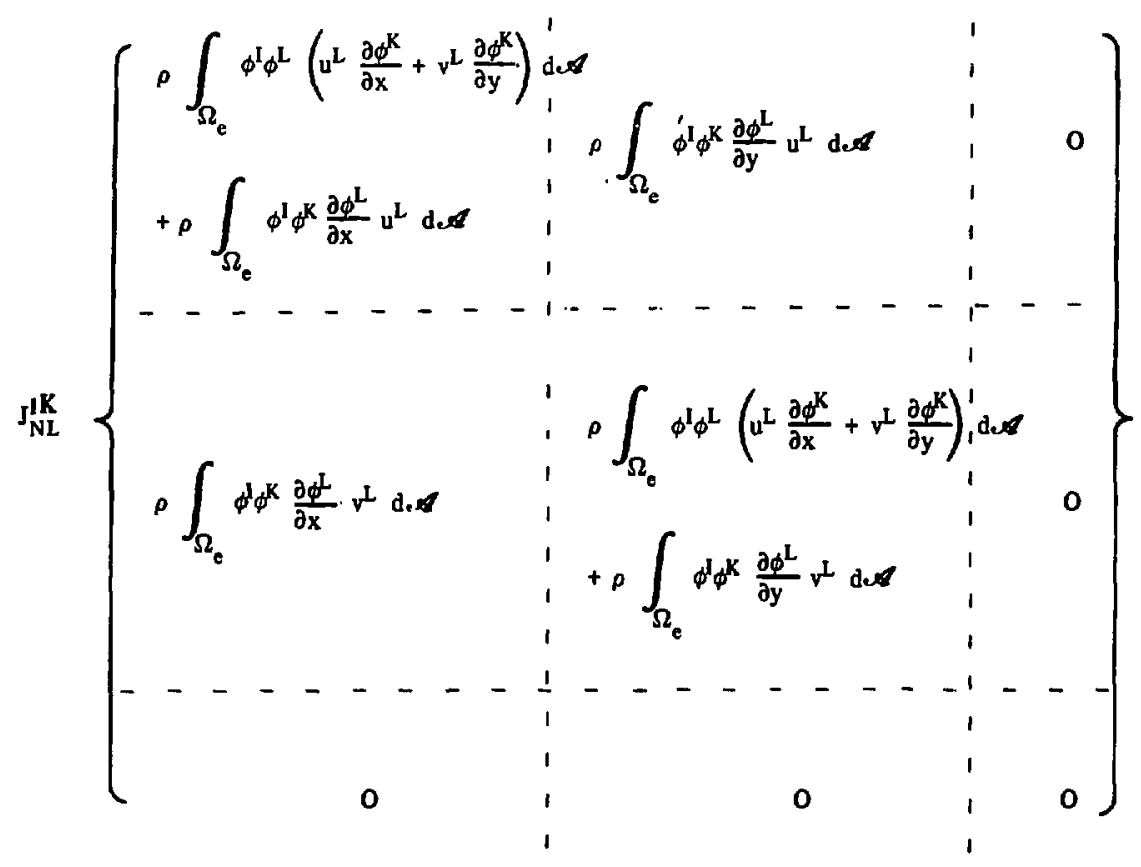

As an alternative to the full Newton-Raphson procedure, we have also experimented with a modified Newton (chord) method wherein $\mathrm{J}_{\mathrm{NL}}$ is updated periodically rather than at every iteration, while the righthand side of Eq. (21) is (necessarily) evaluated at every iteration. This algorithm, although more computationally economical per iteration, converges more slowly than full Newton-Raphson.

\section{Solution Techniques for the Time-Dependent Equations}

For subsequent discussion, we recast the discretized form of the Galerkin Eq. (1f) into the following form:

$$
\begin{aligned}
& \underset{\approx}{\mathbf{u}}+\underset{\approx}{[\mathbf{N}(u)}+\underset{\approx}{\mathbf{K}]} \underset{\sim}{u}+\underset{\approx}{\mathrm{Cp}}=\underset{\sim}{\mathbf{f}} . \\
& \underset{\approx}{\mathbf{T}} \underline{u}=\underline{\sim} .
\end{aligned}
$$

where

$$
\underline{u}=\left\{u^{\mathbf{I}}, v^{\mathbf{I}}\right\}^{\mathbf{T}}
$$

and $\mathrm{K}$ is now the linear matrix consisting of the diffusion terms only. Here $\underset{\mathcal{N}}{\mathrm{K}} \mathrm{Z}$ and $\underset{\approx}{\mathrm{N}}$ are the upper two blocks of $\mathcal{M}_{e}, \underline{K}_{e}$ and $\underline{\sim}_{e}$ in Eqs. (15), (17), (18) respectively, while $\mathcal{E}_{\mathbb{Z}}$ is the remaining first-derivative terms.

Since $M$ is nondiagonal, an explicit time integration algorithm is possible only for cases in which the calculation of $\mathrm{M}$ inverse is practical (i.e., small systems). Problems of this type are of limited interest, however. Some work has been performed (mainly in the structural field) wherein simple but ad hoc mass lumping schemes are employed to "diagonalize" $\mathbf{M}$. Although these lumping schemes have been relatively successful for Lagrangian systems (with second-order time-derivative terms) or pure heat conduction problems, they are often highly inaccurate for large Reynolds number flows or advection-dominated [large $\mathbb{N}(\mathrm{u})$ ] situations. $^{2}$ 
Unless new techniques are developed to handle the mass matrix, it appears that conventional explicit time integration would be impractical.

Unless new techniques are developed to handle the mass matrix, it appears that conventional explicit time integration would be impractical.

We employed, for our transient FEM codes, variable time-step finite difference algorithms with automatic step-size selection based on controlling the local time truncation error. This algorithm utilizes a second order accurate, implicit technique (trapezoid rule) in conjunction with a second order accurate, explicit scheme (Adams-Bashforth). In the calculations, the explicit solution is not actually reported but serves as a predictor for the subsequent implicit (corrector) step, and is also used for computation of the local truncation error. As will be shown later, this particular explicit time algorithm, used in conjunction with the trapezoid rule, does not require inversion of the mass matrix as previously described.

We have successfully tested a similar integration technique on several advection-diffusion calculations. ${ }^{2}$ In particular, for transient problems where steady-state solutions exists, the time-step increments increase very rapidly as steady-state is approached, resulting in computationally economical as well as accurate simulations. (An accurate transient simulation, going all the way steady-state may be accomplished in as few as 50 timesteps.) It is expected that a similar implicit/explicit procedure for time integration might also be useful if finite differences were used for spatial discretization. These basic algorithms are now being applied to the NavierStokes code with the exception that additional schemes are introduced to handle the (implicit) pressure unknown (continuity equation). Briefly, the implicit integration technique involves the steps outlined in the subsequent paragraphs.

\section{Initialization}

To start the problem, we require that the initial velocity field satisfy the condition

$$
\stackrel{c^{T}}{\underset{\sim}{\mathbf{u}}} \mathbf{0}=\underset{\sim}{0} \text {. }
$$

This, in turn, requires the given initial field be a solution of the discretized form of the continuity equation $\left(C^{T_{u_{0}}}=0\right)$. If this condition is not met, the initial violation of mass conversation will immediately lead to "shock" conditions with the resulting numerical noise destroying subsequent accuracy. With a suitably defined initial $u_{0}$ field, we start by solving the following linear system for the initial acceleration and pressure field $\mathrm{P}_{0}$ : (Note that the correct, compatible, initial pressure field can only be obtained in this way.)

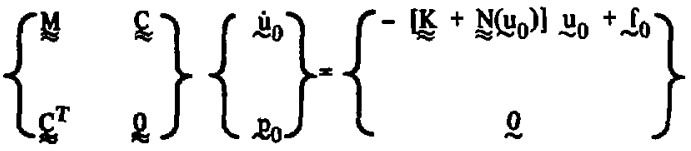

\section{Trapenold Rule}

For the implicit step, we apply the trapezoid rule at time-step $n$ to Eqs. (23) and (24), which results in:

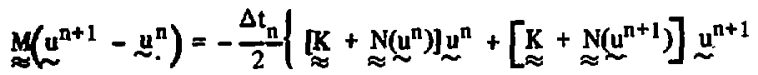

$$
\begin{aligned}
& +\underset{\approx}{C}\left(\underline{p}^{n}+\stackrel{p}{p+1}^{n+1}\right)-\mathfrak{f}_{n}-\mathfrak{f}_{n+1} \mid
\end{aligned}
$$

$$
\stackrel{C}{\mathbf{T}}_{\sim}^{\mathbf{u}^{\mathrm{n}+1}}=\stackrel{0}{\sim}
$$


Upon transposing of terms and multiplying by $2 / \Delta t_{n}$, we obtain a nonlinear algebraic system given symbolicaliy by

$$
A(q) g=\underset{b}{b}
$$

Newton's method applied to this equation gives

$$
J_{m}\left(g_{m+1}^{n+1}-g_{m}^{n+1}\right)=\underset{\sim}{b}-\underset{\approx}{A}\left(g_{m}\right) g_{m}^{n+1}
$$

for the $m+l$ iteration, where

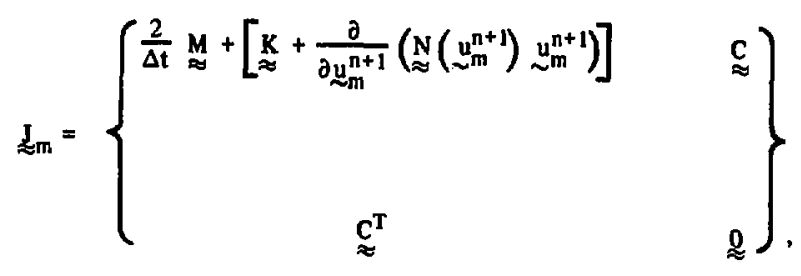

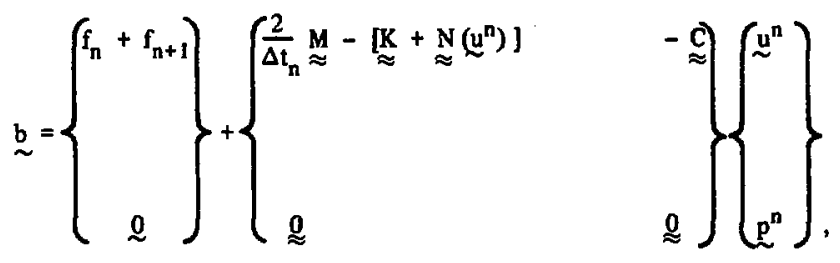

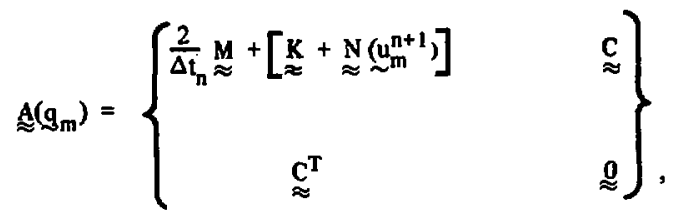

and

$$
q=\left\{\begin{array}{l}
u \\
\sim \\
p
\end{array}\right\} .
$$

For time-dependent problems, where many solutions of the nonlinear algebraic system [Eq. (28)] are required as the time integration proceeds, the cost of updating $\mathrm{J}_{m}$ at every iteration for each time-step (full Newton procedure) would be prohibitive. As an alternative, we are willing to relent and proceed for a number of time steps with an out-of-date Jacobian matrix; a strategy (chord method) which only affects the convergence 
rate, not the final result. Our strategy of Jacobian updates is based on the following simple criteria: (A similar set of criteria has been successfully employed in the sophisticated Gear package developed at LLL. ${ }^{3}$ )

- J is updated whenever the time step $\Delta t$ is increased or decreased (by the selected variable step algorith $\mathrm{m}$ ) by more than about $50 \%$.

- The solution is converging too slowly or is diverging.

The nonlinear system is considered to have converged when the norm of the iterates becomes smaller than $10 \%$ of the local truncation error (discussed below) permitted in one time step.

\section{Adams-Bashforth Expllcit Algorithm and Local Truncnt'sn Erro: Confre!}

We apply a variable step Adams-Bashforth algorithm to the velocity variable (not the pressure, since it is inherently an implicit unknown) as follows:

${\underset{\sim}{n+1}}_{n+1}^{p}={\underset{\sim}{n}}_{n}+\Delta t_{n}\left[\left(1+\frac{\Delta t_{n}}{2 \Delta t_{n-1}}\right) \dot{u}_{n}-\frac{\Delta t_{n}}{2 \Delta t_{n-1}} \stackrel{\sim}{\sim} n-1_{n}\right]$,

where $\dot{b}_{n}=2 / \Delta t_{n-1}\left(u_{n}-u_{n-1}\right)-\dot{u}_{n-1}$ is available from the trapezoid rule result (this is the reason we can employ an explicit algorithm without inverting the mass matrix), and $u_{h+1}$ is the predicted $u$ at time step $n+l$.

The predictor-corrector algorithm at step $n$ is therefore accomplished by first employing AdamsBashforth to generate $\mathcal{u}_{h+1}^{p}$ (predictor step), then using $\mathfrak{u}_{h+1}^{p}$ for the trapezoidal (corrector) step to obtain $u_{n+1}\left[u_{n+1}\right]$ is used for the first guess in $\mathbf{A}\left(\mathrm{q}_{\mathrm{m}}\right) \mathrm{q}_{\mathrm{m}}$ in Eqs. (29) or (32)]. The pressure, at that same time step, is predicted by quadratic extrapolation from calculated values at $n, n-1$, and $n-2$ and corrected during the trapezoid rule portion of the algorithm.

To obtain an expression for the local truncation error, we performed a time truncation error analysis (Taylor series) of both the Adams-Bashforth and trapezoid steps to give, finally

$\dot{d}_{n+1}=\underline{v}_{n+1}-\underline{u}\left(t_{n+1}\right)=\frac{\underline{u}_{n+1}-\underline{u}_{n+1}^{p}}{3\left(1+\frac{\Delta t_{n-1}}{\Delta t_{n}}\right)}+O\left(\Delta t^{4}\right)$,

where $\underline{u}\left(t_{n+1}\right)$ is the exact value of $u$ at $t_{n+1}$ (assuming that $u_{n}$ was exact). In the code, a norm of $g_{n+1}$ is compared with an input local error tolerance parameter $\epsilon$ in order to determine whether time-step changes are required. If a $\Delta t$ change is necessary, we employ the formula

$(\Delta t)_{\text {now }}=0.8(\Delta t)_{\text {old }}\left[\frac{\left(\epsilon V_{\text {max }}\right)}{\left\|d_{n+1}\right\|}\right]^{1 / 3}$,

where a root mean square norm is employed:

$\left\|d_{n+1}\right\|^{2}=\frac{1}{N} \sum_{i=1}^{N}\left[\left(d_{i}\right)_{n+1}\right]^{2}$

$N$ is the total number of velocity variables, and $e V_{\max }$ is an input parameter. Equation (36) reflects the second order accuracy of our time integration scheme [i.e., || $\mathbf{d}|| \sim(\Delta \mathrm{t})^{3}$ ].

Since the time steps are variable, it is necessary to use some interpolation scheme for displaying solutions at any particular output time. We use a consistent quadratic interpolation formula to approximate solution times between two integration time steps.

In an attempt to form some basis of comparison with the variable time-step algorithm, we also developed a constant-time-step integration scheme based on similar ideas but without the truncation error control 
algorithm. For this integration, we have the flexibility of easily incorporating backward-Euler trapezoid rule or any "combination" thereof within the same code.

A finite difference spatial discretization of the Navier-Stokes equations could also benefit from the use of such a time integration scheme, which is both completely stable (A-stable) and is as accurate "as desired" [by means of the selection of $\mathrm{s}$ in Eq. (36)] at all times during a time-dependent calculation. For example, if timedependent boundary conditions are applied that are variable in their transient effects on the flow, the time step would desrease (as necessary) or increase (where possible) to maintain the user-specified time integration error.

\section{STORAGE CONSIDERATIONS AND LINEAR EQUATION SOLVERS}

Following the practice of most FEM codes, we compute element matrix coefficients by means of Gaussian quadrature and store the results at element level sequentially, element-by-element, on disk. In general, we assume all elements to be geometrically different (isoparametric elements) and store a set of coefficients for each element. It is obvious that the amount of information for storage (and later retrieval) can be quite high, even in two dimensions. For example, the present Navier-Stokes formulation requires storage of two sets of coefficients from the nonlinear terms

$$
\int_{\Omega_{\mathrm{e}}} \phi^{\mathrm{I}} \phi^{\mathrm{J}} \frac{\partial \phi^{\mathrm{L}}}{\partial \mathrm{x}} \mathrm{d} \mathscr{\mathcal { C }}, \int_{\Omega_{\mathrm{e}}} \phi^{\mathrm{I}} \phi^{\mathrm{J}} \frac{\partial \phi^{\mathrm{L}}}{\partial \mathrm{y}} \mathrm{d} \mathcal{\alpha}
$$

as well as one set of coefficients from the linear terms. A 9-node (22 degree-of-freedom) element generates two sets of $9 \times 9 \times 9$ entries from the triple-product integrals and a $22 \times 22$ linear matrix. This means that 1942 words of information per element need to be retained on disk. (For our problems, we take some advantage of symmetry and reduce this number to 1063 words per element.)

Several alternatives can be implemented to make the storage problem more tolerable:

1. Use a mesh containing as many similar elements as possible. For example, the meshed region may be regular except for a single layer of elements at each irregular boundary side. In this way, only coefficients for a few different elements are required to be stored either in core or in large core where they can be directly accessed.

2. Since coefficient for the nonlinear terms make up about $70 \%$ of the elemental storage requirement, one might economize by storing only the linear terms and recomputing all nonlinear terms whenever required. This procedure is particularly attractive if reduced integration (say 1-point quadratures) can be tolerated; but in any event is only useful if it leads to overall cost savings.

3. Instead of using the "honest" Galerkin approximation for nonlinear terms, we may choose to use a simpler approximation given by

$$
u v \equiv \sum_{I} u^{\mathbf{I}} v^{I_{\phi}} \phi^{I}
$$

which leads to

$$
\int_{\Omega} \phi^{\mathrm{I}} \mathrm{uvd} \boldsymbol{\omega}=\sum_{\mathrm{J}}\left(\int_{\Omega} \phi^{\mathrm{I}} \phi^{\mathrm{J}} \mathrm{d} \boldsymbol{\varphi}\right) \mathrm{u}^{\mathrm{J}} \mathrm{v}
$$

in the advection terms. Therefore, rather than storing triple subscripted matrices for nonlinear products, we replace these with doubly-subscripted (mass) matrices. The utility of this product approximation has yet to be demonstrated, although initial numerical experiments are mildly encouraging. 
4. Use iterative methods for solving the linear systems. Herative methods are particularly attractive because of low storage requirements.

We have employed a direct (Gaussian elimination) solution techniqus, that uses the frontal algorithm developed by Irons ${ }^{4}$ and Hood ${ }^{5}$ to solve the lineas system of equations. Although the version of the frontal program we employ is practical for solving only small to moderately large systems of equations (say about $<2000$ ), it offers great flexibility in element choice and imposes no bandwidth limitations. It is possible, but usually not desirable, to minimize in-core storage requirements at the expense of many disk transfer operations by using such a frontal solver.

The frontal routine starts by assembling the global matrix from the appropriate ele.nent matrices into core until a preassigned core area is filled. Then a pivotal search (if required) is made from the completely assembled equations (i.e., those equáao.s whose coefficients will no longer change as subsequent element matrices are assembled into nodal equations). These assembled equations are then eliminated with the calculated coefficients placed on disk storage and the original in-core space occupied by these coefficients cleared, ready to accept new equations. When sufficient equations are eliminated, more element information is brought into core and assembled, after which further elimination may proceed as before. After all equations have been eliminated, the solution is obtained by the back-substitution portion of the algorithm.

To accomplish the elimination process for any node-ordering contiguration, the scheme requires a sophisticated bookkeeping algorithm to keep track of equations and their respective elimination sequence. Since elimination may occur for any equation depending on its order of appearance and assembly state, all information related to such an operation needs to be stored and later retrieved during the back-substitution procedure. As a result, the solver may not be very efficient in execution time. Timing studies reveal that the time spent in input/output ( $1 / 0)$ operations is roughly twice as much as that required for central processor unit (CPU) operations. These costs will quickly become intolerable if the problem is much larger (as for a large two-dimensional or for any three-dimensional problems), particularly if many calls to the frorital program are necessary. Other more conventional out-of-core direct solvers, such as those employing profile or banded techniques which take advantage of the matrix structure, may be computationally more efficient (requiring less bookkeeping) but could probably not improve significantly on the I/O problem.

We believe that the matrices generated by reasonably sized three-dimensional or even large twodimensional FEM problems cannot be solved effectively with presently available direct solvers. As an alternative, it appears that iterative solution techniques, with their advantage of low storage requirements, could be ideally suited for problems of the size we envision solving (e.g., 5,000 to 50,000 unknowns). Experience indicates that significantly fewer nodes are required using FEM methods than finite difference methods (FDM); the "work per node" of FEM, however, is significantly greater than that for typical FDM formulations. Although FEM developers have made occasional suggestions that iterative methods may be useful for very large problems, few of the current large codes employ them. The frequently used argument is that they perform unreliably for FEM-type matrices describing complex physical shapes. (Typical finite difference matrices are structurally much simpler.)

The possibility and potential of the successful application of iterative methods in solving FEM problems has not been completely overlooked; however, Seitelman ${ }^{6}$ has shown the potential of a simple iterative method [point Gauss-Seidel and successive overrelaxation (SOR)] with respect to both storage and operation count for two- and three-dimensional problems involving ine Laplacian operatcr. Seitelman further demonstrates that useful results can be expected using a simple combination of direct and iterative methods, wherein the direct solution is applied to the portion of the matrix with small bandwidth; relaxation methods are employed to deal with that portion of the matrix far removed from the diagonal.

Somewhat related techniques have been advocated by Tuff and Jennings ${ }^{7}$ where some of the off-diagonal elements are transposed to the right-hand side for iteration, leaving those elements in the matrix that might lead to convergence problems during the iteration process. A recent two-dimensional, global, barotropic FEM model for Rossby waves ${ }^{8}$ has successfully employed the straightforward, point Gauss-Seidel iteration scheme. Various iterative and direct techniques were employed by Hirsh ${ }^{9}$ in his FEM solution of ocean circulation problems. A new iterative technique employing Tchebychev acceleration techniques on nonsymmetric systems has been developed by Manteuffel ${ }^{10}$ and may hold some promise, at least for steady problems. Pinder ${ }^{11}$ et al., have developed a promising block SOR technique, which they have successfully applied to a wide range of FEM flow problems. They claim significant economic gains over direct methods. Another recent technique, developed by Meijerink and van der Vorst and tested by Kershaw, ${ }^{12}$ utilizes a combined direct/iterative technique called ICCG (Incomplete Cholesky Conjugate Gradient). Yuan, et al., ${ }^{13}$ have experimented with 
point SOR, tlock SOR, and conjugate gradient methods for two-dimensional neutron transport problems and concluded that block SOR is most efficient. Finally, for symmetric problems at least, the conjugate gradient method, as proposed by Fried, ${ }^{14}$ is advocated as being an efficient technique for solving large nonlinear FEM systems.

In our preliminary research, we have performed point-SOR calculations by using a dynamically updated " $\omega$-routine" on the two-dimensional pure advection problem (a "difficult" hyperbolic system with unsymmetric matrices) using our existing FEM code, and were encouraged by the results. (The technique worked well as long as the time step wasn't too large; too large a time step can cause a lack of convergence, as well as a severe loss in accuracy.)

We also tested several iterative algorithms on the Stokes (linear) flow equations. The implementation of iterative schemes for these equations is more difficult because the continuity equations generate zeros on the diagonal entries. After some experimentation, the algorithms that appear to show the most promise are those where the continuity constraint is relaxed via a penalty technique; similar to a technique proposed by Chorin ${ }^{15}$ for solving incompressible flow problems using finite difference methods.

Thus, instead of attacking [Eq. (7)], we replace the continuity equation by

$$
-\varepsilon \frac{\partial p}{\partial t}=\nabla \cdot V
$$

In the limit $\varepsilon \rightarrow 0$, we recover the original continuity equation. Also, it is anticipated that if $\varepsilon$ is small enough, we generate a solution where Eq. (7) is closely approximated. For problems with steady-state solutions, continuity will be satisfied for any $\varepsilon$, since ap/at $\rightarrow 0$ in the steady-state limit.

There is yet another version of the penalty method (Hughes, et al. ${ }^{16}$ ), potentially applicable to iterative solution methods. In this technique, the pressure variable is eliminated completely from the set of unknowns; here, the continuity equation is replaced by

$$
p=-\lambda \nabla \cdot v
$$

where $\lambda$ is the penalty parameter, taken, in this case, to be very "large". Upon substituting Eq. (38) into Eq. (8), the pressure variable is eliminated from Eq. (6), and Eq. (7) is discarded. We may then, when necessary for output purposes, recover the pressure (as needed) once $V$ is obtained, through Eq. (38). The described method has been successfully applied to both steady-state and transient problems. ${ }^{16,17}$

We anticipate that a successful iterative scheme can be developed based on some variant of the described penalty function approaches. In the implementation of the scheme, we will compute global integrals elementby-element as before, but only once at the beginning of a problem. Entries for the global matrix will then be formed on one equation (or block) at a time as required for iterative processing. The equations are then blocked in such a way as to obtain a block tridiagonal structure. The off-diagonal blocks may be treated via SOR, while the subsystems of diagonal blocks will be solved successively by employing conventional (banded) direct techniques.

A key feature of any iterative method, of course, is that only non-zero matrix coefficients will be stored and manipulated. At this point, the major uncertain, but critical, feature is the effect of the "penalty parameter" on the convergence of the iteration process (the "best" iterative scheme is, of course, also uncertain). For transient problems it is highly desirable that the iterative method can converge for time steps that are based on accuracy requirements (via local time truncation error control in our case) rather than on iterative convergence requirements. For sufficiently small time steps, the convergence of SOR (for example) is probably assured, owing to the symmetric, positive-definite character of the mass matrix. The hope is that the method will still converge at a satisfactory rate for larger time steps. 


\section{ACKNOWLEDGMENTS}

We are indebted to the following people for their contributions to this project: Prof. R. L. Sani, for his work in the areas of theoretical formulation and programming logic; Dr. A. C. Hindmarsh for his assistance in the development of the strategy for time integration and in the areas of linear and nonlinear algebra; Dr. N. K. Madsen for his implementation of several iterative solution techniques; and finally, to T. S. Stullich for his overall programming assistance.

\section{REFERENCES}

1. P. S. Huyakorn, C. Taylor, R. L. Lee, and P. M. Gresho, "A Comparison of Various MixedInterpolation Finite Elements in the Velocity-Pressure Formulation of the Navier-Stokes Equations," to appear in Computer and Fluids.

2. P. M. Gresho and R. L. Lee, "Advection-Dominated Flows with Emphasis on the Consequences of Mass Lumping," to appear in Finite Elements in Fluids, Vol. 3., J. Wiley \& Sons (1978).

3. A. C. Hindmarsh, GEAR: Ordinary Differential Equation Solver, Lawrence Livermore Laboratory, Rept. UCID-30001, Rev, 3 (1974).

4. B. M. Irons, "A Frontal Solution Program for Finite Element Analysis," Int. J. Num. Meth. Engng. 2, 5-32 (1970).

5. P. Hood, "Frontal Solution Program for Unsymmetric Matrices," Int. J. Num. Meth. Engng. 10, 379-399 (1976).

6. L. H. Seitelman, "Some Practical Solution Techniques for Finite Element Analysis," in The Mathematics of Finite Elements and Applications (Academic Press, New York, 1973).

7. A. D. Tuff and A. Jennings, "An Iterative Method for Large Systems of Linear Structural Equations," Int. J. Num. Meth. Engng. 7, 175-183 (1973).

8. D. E. Hinsman, Application of a Finite Element Method to the Baratiopic Primitive Equations, M. S. thesis, Naval Postgraduate School, Monterey, California (1975).

9. J. A. Hirsch and W. L. Bryes, Sparse Matrix Problems in a Finite Element Open Ocean Model, Rept. TR23-75, Div. of Engng. and Appl. Phys., Harvard University (1975).

10. T. A. Manteuffel, "The Tchebyche:- Iteration for Nonsymmetric Linear Systems", Num. Math. 28, 307 (1977).

11. G. F. Pinder, G. McCracken, C. Voss, and M. Ungo, Block Iterative Finite Element Preprocessed Scheme (BIFEPS)-A Package for Simulation of Nonlinear Transient Problems with One or Two Governing Equations in Two or Three Dimensions, Princeton University, Rept. 77-WR-12 (1977).

12. D. Kershaw, The Incomplete Cholesky-Conjugate Gradient Method for the Iterative Solution of Systems of Linear Equations, Lawrence Livermore Laboratory, Rept. UCRL-78333 (1976).

13. Y. C. Yuan, E. E. Lewis, and W. F. Miller, Jr., "Iterative Solution Methods for Two-Dimensional Finite Element Approximations in Neutron Transport," Proc. of the Conf. of Comp. Meth. in Nuclear Energy III. Charleston, S.C., 85-100 (1975).

14. I. Fried, "A Gradient Computational Procedure for the Solution of Large Problems Arising from the Finite Element Discretization Method", Int. J. Num. Meth. Engng. 2, 477-494 (1970).

15. A. J. Chorin, "A Numerical Method for Solving Incompressible Viscous Flow Problems," J. Comp. Phys. 2, 12-26 (1967).

16. T. J. R. Hughes, R. L. Taylor, and J. F. Levy, "A Finite Element Method for Incompressible Flows," preprints of the Second International Symposium of Finite Elements in Flow Problems, Rapallo, Italy, 1-15 (1976).

17. M. Bercovier and M. Engelman, "A Finite Element for the Numerical Solution of Viscous Incompressible Flows," paper submitted to $J$. Comp. Phys. 